\title{
Nearly Injective Semimodules
}

\author{
Sarah H. A. Alsaebari \\ Asaad M. A. Alhossaini \\ Department of Mathematics, College of Education for pure Sciences, University of \\ Babylon
}

sarah1993100@gmail.com

asaad_hosain@itnet.uobabylon.edu.iq

\begin{tabular}{l}
\hline ARTICLE INFO \\
\hline Submission date: $10 / 7 / 2018$ \\
Acceptance date: $6 / 9 / 2018$ \\
Publication date: $10 / 3 / 2019$
\end{tabular}

\begin{abstract}
An injectivity in the category of semimodules over semiring was studied by many authors recently. On the other hand, the concept of injectivity, in the category of modules over ring, was generalized in many different directions. In particular, injective modules relative to preradical were some of those generalizations.

As an analogue to module theory, in this paper, we introduce and investigate the notion of "injective semimodule relative to Jacobson radical (namely nearly injective semimodule)".

Key words: subtractive semimodule; Jacobson radical; nearly injective semimodule; nearly direct summand; nearly split homomorphism.

\section{Introduction}

Throughout this work, Ş stands for a commutative semiring with identity and a semimodule means a unitary left Ş-semimodule. An Ş-subsemimodule $L$ of an Şsemimodule $A$ is called subtractive if for all $a, a^{\prime} \in A, a+a^{\prime}, a \in L$ implies $a^{\prime} \in L$, it is clear that 0 and $A$ are subtractive Ş-subsemimodules of $A$. An Ş-semimodule $A$ is a subtractive Ş-semimodule if it has only subtractive subsemimodules [1]. For convince all Ş-semimodules assumed in this work will be considered subtractive.
\end{abstract}

An Ş-semimodule $W$ is called injective if for every Ş-monomorphism $\alpha: A \rightarrow B$ and for each Ş-homomorphism $\beta: A \rightarrow W$, there is an Ş-homomorphism $h: B \rightarrow W$ such that $h \alpha=\beta[1]$.

In 2000, nearly injective modules were discussed in [2] as a generalization of injective modules. A module $W$ is called nearly injective, if for every monomorphism $\alpha: A \rightarrow B$ (where $A$ and $B$ are two modules) and for each homomorphism $\beta: A \rightarrow W$, there is a homomorphism $h: B \rightarrow W$ such that $(h \circ \alpha)(x)-\beta(x) \in J(W), \forall x \in A$ where $J(W)$ is the Jacobson radical of the module $W$, which is defined to be the intersection of all maximal submodules of $W$.

As an analogue to the case in modules, nearly injective semimodule, is introduced in this work. An Ş-semimodule $W$ is called nearly injective, if for every Smonomorphism $\alpha: A \rightarrow B$ (where $A$ and $B$ are two Ş-semimodules), each Ş- 
homomorphism $\beta: A \rightarrow W$, there is an Ş-homomorphism $h: B \rightarrow W$ such that $\pi_{J} h \alpha=\pi_{J} \beta$, where $\pi_{J}: W \rightarrow W / J(W)$ is the natural epimorphism, and $J(W)$ is the Jacobson radical of $W$. As in modules $J(W)$ is the intersection of all maximal Şsubsemimodules of $W$.

Many properties and characterizations of nearly injectivity were investigated. The main results of this work are: It is shown that these semimodules are closed under arbitrary direct product, finite direct sum and direct summand. Nearly direct summand with proof that an Ss-semimodule $W$ is nearly injective if and only if, it is a nearly direct summand of every extension of itself. Therefore the nearly split homomorphism with proof that an Ş-semimodule $W$ is nearly injective if and only if, for each Şsemimodule $F$, every Ş-monomorphism $\alpha: W \rightarrow F$ is nearly split.

In addition to section 1, there are two sections. Section 2 consists the preliminaries that are needed in the investigations. Some of these were found in the literatures. In section 3, injective Ş-semimodule and their properties and characterizations were given.

\section{2- Preliminaries}

In this section same definitions, their properties and characterizations of these Şsemimodules needed in this work.

Definition 2.1 [3]. Let Ş be a semiring. A left Ş-semimodule $A_{S}$ is a commutative monoid $(A,+, 0)$ for which we have a function $S \subset \times A \rightarrow A$, defined by $(s, a) \mapsto s a$ such that $\forall s, t \in S$ and $a, a^{\prime} \in A$,

1. $s\left(a+a^{\prime}\right)=s a+s a^{\prime}$.

2. $(s+t) a=s a+t a$.

3. $(s t) a=s(t a)$.

4. $0_{S} a=0_{A}=s 0_{A}$.

If $1_{\widehat{S}} a=a$ holds for each $a$ in $A$ then a left Ş-semimodule $A$ is called unitary.

Definition 2.2 [3]. Let $L$ be a subset of a left Ş-semimodule $A$ then $L$ is called subsemimodule of $A$ if $L$ is closed under addition and scalar multiplication. In this case it is denoted by $L \hookrightarrow A$.

Definition 2.3 [3] An Ş-subsemimodule $L$ of an Ş-semimodule $A$ is called subtractive if for all $a, a^{\prime} \in A \quad a, a+a^{\prime} \in L$ implies $a^{\prime} \in L$.

An Ş-semimodule $A$ is a subtractive Ş-semimodule if it has only subtractive subsemimodules.

Definition 2.4 [4] An Ş-semimodule $A$ is called yoked if for all $a, b \in A$ there exists $c \in A$ such that $a=b+c$ or $a+c=b$.

Definition 2. 5 [4] An Ş-semimodule $A$ is additively cancellative if $a+c=b+c \Rightarrow$ $a=b$. 
We denote to the Ş-semimodule that possess the three conditions, yoked, cancellative and subtractive by $\mathcal{Y} \mathcal{C} \mathcal{S}$-semimodule.

Definition 2.6 [5] An Ş-subsemimodule $L$ of an Ş-semimodule $A$ is called a direct summand of $A$ if there exists Ş-subsemimodule $K$ such that $A=L \oplus K$ and $A$ is called a direct sum of $L$ and $K$.

Definition 2.7 [3] Let $A$ and $B$ be Ş-semimodules. A homomorphism from $A$ to $B$ is a map $\varphi: A \rightarrow B$ such that

1. $\varphi\left(a+a^{\prime}\right)=\varphi(a)+\varphi\left(a^{\prime}\right)$ and

2. $\varphi(s a)=s \varphi(a) \quad \forall a, a^{\prime} \in A$ and $s \in S$.

For a homomorphism of Ş-semimodules $\varphi: A \rightarrow B$ we define:

1. $\operatorname{ker}(\varphi)=\{a \in A \mid \varphi(a)=0\}$.

2. $\varphi(A)=\{\varphi(a) \mid a \in A)\}$.

3. $\operatorname{Im}(\varphi)=\left\{b \in B \mid b+f(a)=f\left(a^{\prime}\right)\right.$ for some $\left.a, a^{\prime} \in A\right\}$.

A homomorphism of Ş-semimodules $\varphi: A \rightarrow B$ is a:

1. monomorphism, if for any Ş-semimodule $L$ and Ş-hhomomorphism's $\alpha \beta: L \rightarrow A$ with $\varphi \circ \alpha=\varphi \circ \beta$, we have $\alpha=\beta$.

2. epimorphism, if for any Ş-semimodule $K$ and Ş-homomorphisms $\alpha, \beta: B \rightarrow K$ with $\alpha \circ \varphi=\beta \circ \varphi$, we have $\alpha=\beta$.

3. isomorphism if $\varphi$ is monomorphism and epimorphism.

4. image regular ( $i$-regular), if $\varphi(A)=\operatorname{Im}(\varphi)$.

5. kernel regular (1?-regular) if $\varphi(a)=\varphi\left(a^{\prime}\right) \Rightarrow a+k=a^{\prime}+k^{\prime}$ for some, $k, k^{\prime} \in$ $\operatorname{ker}(\varphi)$.

6. regular if $\varphi$ is $i$-regular and ?-regular.

Definition 2.8 [3] The (possibly infinite) sequence of $S$-semimodules

$\ldots A_{i} \stackrel{\varphi}{\rightarrow} A_{i+1} \stackrel{\varphi_{i+1}}{\longrightarrow} A_{i+2} \stackrel{\varphi_{i+2}}{\longrightarrow} \ldots$ is said to be:

1. exact, if $\operatorname{Im} \varphi_{i}=\operatorname{ker} \varphi_{i+1} \forall i \in I$.

2. proper exact, if $\varphi_{i}\left(A_{i}\right)=\operatorname{ker} \varphi_{i+1} \forall i \in I$.

Proposition 2.9 [3] Let $A$ and $B$ be Ş-semimodules, then a homomorphism of Şsemimodules $\varphi: A \rightarrow B$ is:

1. injective if and only if it is a monomorphism.

2. surjective if and only if it is epimorphism and $\varphi(A) \subseteq B$ is subtractive.

Lemma 2.10 [3] Let $A, B$ be Ş-semimodules and $\varphi \in \operatorname{Hom}_{S}(A, B)$, then

1. $\operatorname{Im}(\varphi)$ is subtractive.

2. $\varphi(A)$ is subtractive if and only if $\varphi(A)=\operatorname{Im}(\varphi)$. 
The following two lemmas and corollary had been proved for modules (see e.g. [6, pp.60-61]), but for semimodules they need extra conditions and then new converted proofs that were not found in the literatures.

Lemma 2.11 Let $A$ and $B$ be $\mathcal{Y C S}$-semimodule and $\varphi \in \operatorname{Hom}_{\widehat{S}}(A, B)$ be an Şhomomorphism then,

1. if $L \hookrightarrow A \Rightarrow \varphi^{-1}(\varphi(L))=L+\operatorname{ker}(\varphi)$.

2. if $K \hookrightarrow B \Rightarrow \varphi\left(\varphi^{-1}(K)\right)=K \cap \operatorname{Im}(\varphi)$.

Proof: (1) Let $x \in \varphi^{-1}(\varphi(L)) \Rightarrow \varphi(x) \in \varphi(L) \Rightarrow \varphi(x)=\varphi(l) \quad l \in L$.

Then, either $x=l+k$.

$\Rightarrow \varphi(l)+\varphi(k)=\varphi(l) \Rightarrow \varphi(k)=0 \Rightarrow k \in \operatorname{ker}(\varphi) \Rightarrow x \in L+\operatorname{ker}(\varphi)$.

Or $x+k=l \Rightarrow \varphi(x)=\varphi(x)+\varphi(k) \Rightarrow \varphi(k)=0 \Rightarrow k \in \operatorname{ker}(\varphi)$

$\Rightarrow l \in L+\operatorname{ker}(\varphi) \wedge k \in L+\operatorname{ker}(\varphi) \Longrightarrow x \in L+\operatorname{ker}(\varphi)$, by subtractive property.

Thus $x \in L+k e r(\varphi)$ (in any case). The other direction is clear.

(2) From set theory $\varphi\left(\varphi^{-1}(K)\right)=K \cap \varphi(A)$, by subtractive property $\varphi(A)=\operatorname{Im}(\varphi)$.

Lemma 2.12 Let $A$ and $B$ be $\mathcal{Y} \mathcal{C} \mathcal{S}$-semimodule and the following diagram be commutative

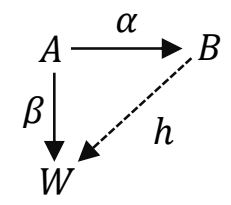

(i.e. $\beta=h \alpha$ ) then,

1. $\operatorname{Im}(\alpha)+\operatorname{ker}(h)=h^{-1}(\operatorname{Im}(\beta))$

2. $\operatorname{Im}(\alpha) \cap \operatorname{ker}(h)=\alpha(\operatorname{ker}(\beta))$

Proof: (1) let $\beta=h \alpha \Rightarrow \operatorname{Im}(\beta)=\operatorname{Im}(h \alpha)=h(\operatorname{Im}(\alpha))$

$\Rightarrow h^{-1}(\operatorname{Im}(\beta))=h^{-1}(h(\operatorname{Im}(\alpha)))=\operatorname{Im}(\alpha)+\operatorname{ker}(h)$ by $\operatorname{Lemma}(2.11(1))$.

(2) Let $\operatorname{ker}(\beta)=\operatorname{ker}(h \alpha)=\alpha^{-1}(\operatorname{ker}(\beta))$.

$\Rightarrow \alpha(\operatorname{ker}(\beta))=\alpha\left(\alpha^{-1}(\operatorname{ker}(\beta))\right)=\operatorname{Im}(\alpha) \cap \operatorname{ker}(h)$ by Lemma(2.11(2)). 
Corollary 2.13 Let $A$ and $B$ be $\mathcal{Y C} \mathcal{S}$-semimodule and the following diagram be commutative

(i.e. $h \alpha=\beta$ ) then,

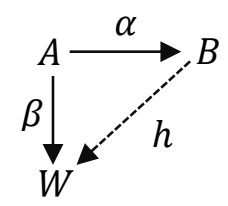

1. $\beta$ is epimorphism $\Rightarrow \operatorname{Im}(\alpha)+\operatorname{ker}(h)=B$.

2. $\beta$ is monomorphism $\Rightarrow \operatorname{Im}(\alpha) \cap \operatorname{ker}(h)=0$.

3. $\beta$ is isomorphism $\Rightarrow \operatorname{Im}(\alpha) \oplus \operatorname{ker}(h)=B$.

Proof: consequently from Lemma (2.12).

Definition 2.14 [1] An Ş-semimodule $A$ is called injective if for every Şmonomorphism $\alpha: A \rightarrow B$ and for each Ş-homomorphism $\beta: A \rightarrow W$, there is an Şhomomorphism $h: B \rightarrow W$ such that the following diagram is commutative

(i.e. $h \alpha=\beta$ ).

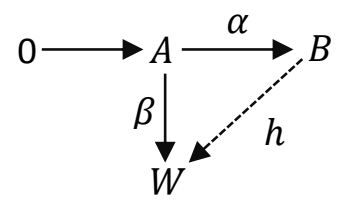

Definition 2.15. [1]. An Ş-semimodule $A$ is called ?-injective if for every Şmonomorphism $\alpha: A \rightarrow B$ and for each Ş-homomorphism $\beta: A \rightarrow W$, there is a ?regular Ş-homomorphism $h: B \rightarrow W$ such that the following diagram be commutative

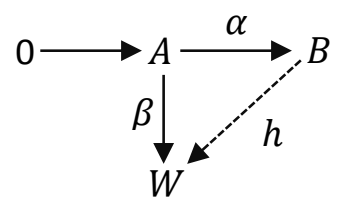

(i.e. $h \alpha=\beta$ ).

Clearly if $W$ is called ?-injective then $W$ is injective.

Proposition 2.16. [1] Let $\left\{W_{\lambda}\right\}_{\lambda \in \Lambda}$ be a family of Ş-semimodules then $\prod_{\lambda \in \Lambda} W_{\lambda}$ is injective if and only if each $W_{\lambda}$ is injective.

Proposition 2.17. Let $\left\{W_{\lambda}\right\}_{\lambda \in \wedge}$ be a family of Ş-semimodules and $W=\oplus_{\lambda \in \Lambda} W_{\lambda}$, then:

1. If $W$ is injective then each $W_{\lambda}$ is injective.

2. If each $W_{\lambda}$ is injective and $\Lambda$ is finite set then $W$ is injective. 
Proof: (1) The proof is similar to the proof of the necessity part of Proposition (2.16).

(2) Clear. Since the product and coproduct coincide in the finite case, and by Proposition(2.16).

Definition 2.18.[7]. Let $W$ be an Ş-semimodule. An Ş-subsemimodule $A$ of $W$ is called large (essential) Ş-subsemimodule of $W$ if for every S-subsemimodule $U$ of $W$ , $A \cap U=0$ implies $U=0$, in this case we say that $W$ is an essential extension of $A$. $W$ is called maximal essential extension of $A$ if whenever $L$ is a proper extension of $W$ then $L$ is not an essential extension of $A$. Note that we shall denote the statement " $A$ is a large subsemimodule $A$ of the Ş-semimodule $W$ " by $A \preccurlyeq_{e} W$.

Definition 2.19. An injective Ş-semimodule $W$ is called minimal injective extension of an SS-subsemimodule $A$ if $W$ is an extension of $A$ and whenever $L$ is a proper subsemimodule of $W$ which contains $A$ then $L$ is not injective.

The following statement is true for any module, see for example [6, pp. 114], but for semimodules the subtractive condition is needed and we have to give a corresponding proof.

Lemma 2.20. Let $W$ be a subtractive Ş-semimodule. If $A$ is a subsemimodule of $W$ and $B$ is a subsemimodule of $W$ maximal with the property $A \cap B=0$, then $A \oplus B \preccurlyeq_{e} W$.

Proof: Assume that both $A$ and $B$ are subsemimodules of the subtractive SSsemimodule $W$ and $B$ is maximal with the property $A \cap B=0$. Then it is clear that $A+B=A \oplus B$. If $C$ is a subsemimodule of $W$ and $(A+B) \cap C=0$, then we have $A \cap C=0$ and $B \cap C=0$. We claim that $A \cap(B+C)=0$. For if $a \in A \cap(B+$ $C)=0$, then $a=b+c$ with $a \in A, b \in B$ and $c \in C$.

Now, $a \in A$ implies $a \in A+B$ and $b \in B$ implies $b \in A+B$. Since $A+B$ is subtractive, we must have $c \in A+B$, so $c \in(A+B) \cap C)=0$, that is $c=0$. Hence $a=b \in A \cap B=0$.

By maximality of $B$, it follows $B+C=B$ and since $B \cap C=0$, we have $C=0$.

Therefore $A \oplus B \preccurlyeq_{e} W$.

Note: Analogue to the case in modules, such subsemimodule $B$ (with the property given in the previous lemma will be called an intersection complement of $A$ in $W$ (shortly inco of $A$ in $W$ ), see [6, D. 5.2.1]. As in the proof above an inco of a given subsemimodule, always exists.

Proposition 2.21. Let $W$ be a $\mathcal{Y C} \mathcal{S}$-semimodule and contained in an injective Şsemimodule then $W$ is injective if and only if it is a direct summand of every extension of itself. 
Proof: Suppose that $W$ is injective and $A$ is a proper extension of $W$, consider the following diagram. Where $\alpha$ is a monomorphism

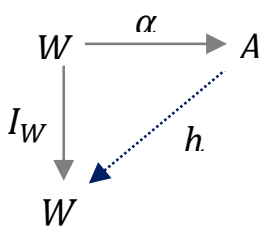

Such that $h \alpha=I_{W} \Rightarrow \operatorname{Im}(\alpha)$ is direct summand in $A$ (Corollary (2.13)).

Since $\alpha$ is a monomorphism, $W \cong \operatorname{Im}(\alpha)$, hence $W$ is direct summand in $A$.

Conversely, suppose that $W$ is direct summand of every extension of it.

Now, since $W$ is contained in an injective Ş-semimodule then $W$ has an injective extension Ş-semimodule. say $L$. Thus $W$ will be a direct summand of $L$ and so will be injective by proposition (2.16).

Proposition 2.22. Let $W$ be an essential extension of $A$ and let $B$ an injective extension of $A$ then the inclusion mapping of $A$ into $B$ can be extended to an embedding of $W$ in $B$.

Proof: The same proof as in the case of modules (see [8, pp.41]).

Proposition 2.23. Let $A$ be an Ş-semimodule and $B$ an injective extension of $A$ then $B$ has subsemimodule $W$ which is a maximal essential extension of $A$.

Proof: The same proof as in the case of modules (see[8, pp.42]).

The proof of the following Theorem (2.24) and Proposition (2.25) are similar to the case of modules, by considering extra condition that it is needed for semimodules (see[8, pp.43]). For completeness we give a full proof.

Theorem 2.24. Let $W$ be a $\mathcal{Y C S}$-semimodule and contained in an injective Şsemimodule, then $W$ is injective if and only if it has no proper essential extension.

Proof: Suppose that $W$ is injective and let $L$ be a proper extension of $W$.

Now, by Proposition (2.21) $W$ is direct summand of $L$, so it cannot be essential in $L$.

Conversely, suppose that $W$ has no proper essential extension.

Now, let $L$ be any extension of $W$, and Let $K$ be an inco of $W$ in $L$. (we assume that $L$ is a proper extension of $W)$. Then $L / K \supseteq(W \oplus K) / K \cong W$ (by the isomorphism theorem see [6]). 
That is $L / K$ is an extension of $W$, and so by assumption $W$ is not essential in $L / K$ $((W \oplus K) / K$ is not essential in $L / K)$. Then there exists $Y \subseteq L$ such that $Y / K \cap$ $(W \oplus K) / K=0$ which implies $Y \cap(W \oplus K)=K$.

Hence $Y \cap W \subseteq K \cap W=0 \Rightarrow Y \cap W=0 \wedge Y \supseteq K$.

Which contradicts the maximality of $K$. So, $L=W \oplus K$.

Since $L$ is an arbitrary extension of $W$, thus $W$ is a direct summand of any extension of it.

Therefore by Proposition (2.21) $W$ is injective.

Proposition 2.25. Let $W$ be a $\mathcal{Y C} \mathcal{S}$-semimodule which is contained in an injective Ssemimodule. If $A$ is an Ş-semimodule contained in $W$, then the following statements are equivalent:

1. $W$ is an essential injective extension of $A$.

2. $W$ is a maximal essential extension of $A$.

3. $W$ is a minimal injective extension of $A$.

Proof: $(1) \Leftrightarrow(2)$ is obvious from Theorem (2.24).

$(2) \Longrightarrow(3)$. Assume, $W$ is a maximal essential extension of $A$. Then $W$ must be injective by Theorem (2.24).

Let $U$ be an injective extension of $A$ contained in $W$. Then $W$ is an injective extension of $U$, so $W=U$ by Theorem (2.24) applied to $U$.

Hence $W$ is a minimal injective extension of $A$.

(3) $\Longrightarrow(1)$ Assume, $W$ is a minimal injective extension of $A$.

Now, by Proposition (2.23) $W$ has a subsemimodule $L$ which is a maximal essential extension of $A$ and so injective it follows that $L=W$ and (1) is established.

An Ş-semimodule $W$ satisfying the conditions of Proposition (2.25) is called an injective envelope (or injective hull) of $A$ (if it exists), we use the notation $E(A)$ to stand for an injective envelope of $A$ [9].

Recall that a maximal Ş-subsemimodule of a semimodule $A$ is a subsemimodule of $A$ that is not contained properly in any other proper subsemimodule of $A$.

Definition 2.26[10]. Let $W$ be an Ş-semimodule and $A$ be a non-zero Şsubsemimodule of $W$. We say that $A$ is a small (superfluous ) S-subsemimodule of $W$ if for every S-subsemimodule $U$ of $W, A+U=W$ implies $U=W$. Then we shall denote a small Ş-subsemimodule $L$ of Ş-semimodule $A$ by $L \preccurlyeq_{S} A$. 
Definition 2.27[10]. Let $A$ be an Ş-semimodule, the Jacobson radical of $A$ is denoted by $J(A)$ and defined as

$$
J(A)=\bigcap_{L \subseteq A}\{L: L \text { is a maximal subsemimodule of } A\} .
$$

Proposition 2.28[10]. Let $A$ be an Ş-semimodule, then

$$
\sum_{L \aleph_{s} A} L=\bigcap_{M \subseteq A}\{M: \text { is a maximal subsemimodule of } A\} .
$$

Proposition 2.29[10]. Let $A$ be an Ş-semimodule and $J$ is a Jacobson radical of $A$ then:

1. $J(A) \hookrightarrow A$. In particular,

2. Let $A$ and $B$ be subtractive Ş-semimodules and $\varphi \in \operatorname{Hom}_{S}(A, B)$ be ?] -regular Şhomomorphism ( $\varphi$ is an epimorphism and ?-regular), then $\varphi(J(A)) \subseteq J(B)$, in particular if $L \hookrightarrow A$ then $J(L) \hookrightarrow J(A)$.

3. $J(A / J(A))=0$.

Note: The Proposition (2.29(2)) is true when $\varphi$ be an Ş-monomorphism (see[11]).

\section{3- Nearly injective Ş-semimodules:}

Definition 3.1. An Ş-semimodule $W$ is called nearly injective, if for every Şmonomorphism $\alpha: A \rightarrow B$ (where $A$ and $B$ are two Ş-semimodules), each Şhomomorphism $\beta: A \rightarrow W$, there is an S-homomorphism $h: B \rightarrow W$ such that $\pi_{J} h \alpha=\pi_{J} \beta$, where $\pi_{J}: W \rightarrow W / J(W)$ is the natural epimorphism and $J(W)$ is the Jacobson radical of $W$.

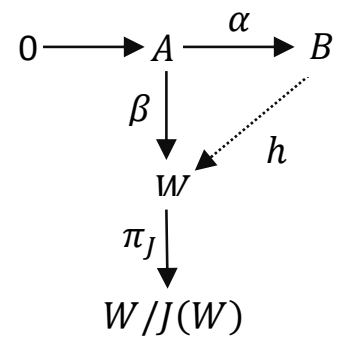

\section{Examples and Remarks 3.2.}

1. every injective semimodule is nearly injective.

2. every ? -injective semimodule is nearly injective.

3. Every Ş-semimodule which has no maximal subsemimodule is nearly injective.

4. It is clear that if $J(W)=0$ then $W$ is injective if and only if it is nearly injective.

5. If $W_{1} \cong W_{2}$, and $W_{1}$ is nearly injective then $W_{2}$ is nearly injective, too.

6. 
Proposition 3.3. An Ş-semimodule $W$ is nearly injective if and only if, for any diagram

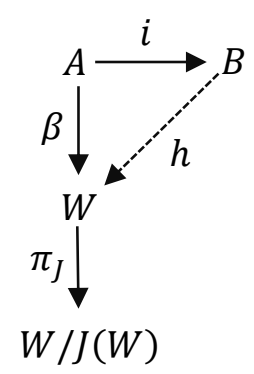

(where $A$ is an S-subsemimodule of $B$ and $i$ is the inclusion mapping) there exists an Ş-

homomorphism $h: B \rightarrow W$ such that $\pi_{J} h i=\pi_{J} \beta$, where $\pi_{J}: W \rightarrow W / J(W)$ is the natural epimorphism.

Proof: The necessity is clear.

Sufficiency: consider the following diagram

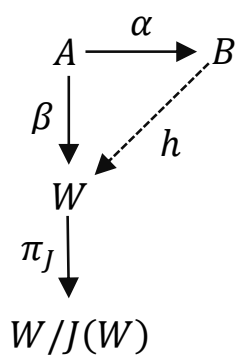

(with $\alpha$ is an Ş-monosophism and $\beta$ is homomorphism).

Now, we define $\varphi: A \rightarrow \alpha(A)$ that is $i \varphi=\alpha$ (where $i: \alpha(A) \rightarrow B$ is the inclusion mapping).

Thus we have the following diagram.

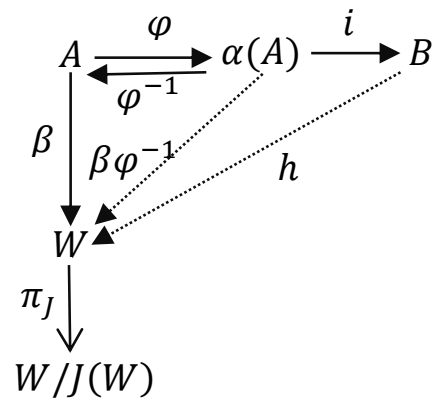

Then by supposition there exists an Ş-homomorphism $h: B \rightarrow W$ such that $\pi_{J} \beta \varphi^{-1}=$ $\pi_{J} h i$

$\Rightarrow \pi_{J} \beta \varphi^{-1} \varphi=\pi_{J} h i \varphi \Rightarrow \pi_{J} \beta=\pi_{J} h \alpha$. Hence $W$ is nearly injective. 
Proposition 3.4. Let $W$ be an Ş-semimodule, then the following statements are equivalent:

1. $W$ is nearly injective Ş-semimodule.

2. For every diagram,

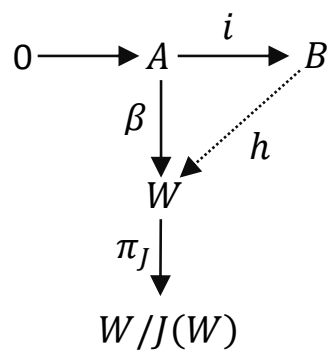

Where $i$ is the inclusion map and $\beta$ is any homomorphism, there exists an Shomomorphism $h: B \rightarrow W$ such that $\pi_{J} h i=\beta \pi_{J}$, where $\pi_{J}: W \rightarrow W / J(W)$ is the natural epimorphism.

3. For every diagram with $A \preccurlyeq_{e} B$,

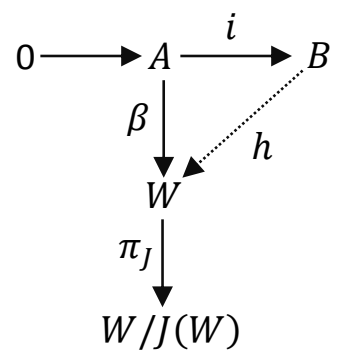

There exists an Ş-homomorphism $h: B \rightarrow W$ such that $\pi_{J} h i=\beta \pi_{J}$, where $\pi_{J}: W \rightarrow$ $W / J(W)$ is the natural epimorphism.

Proof: (1) $\Leftrightarrow$ (2) is by Proposition (3.3) (2) $\Rightarrow$ (3) is obvious.

$(3) \Rightarrow(1)$, given any diagram with exact row, and $\beta: A \rightarrow W$ be any Şhomomorphism.

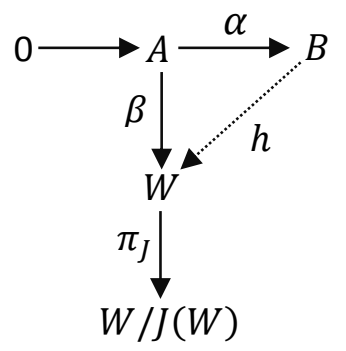

Let $\varphi: A \rightarrow \alpha(A)$ defined by $\varphi(a)=\alpha(a)$ for all $a$ in $A$, then $\varphi$ is an isomorphism, $i \varphi=\alpha$, where $i: \alpha(A) \rightarrow B$ is the inclusion map. Consider the following diagram, 


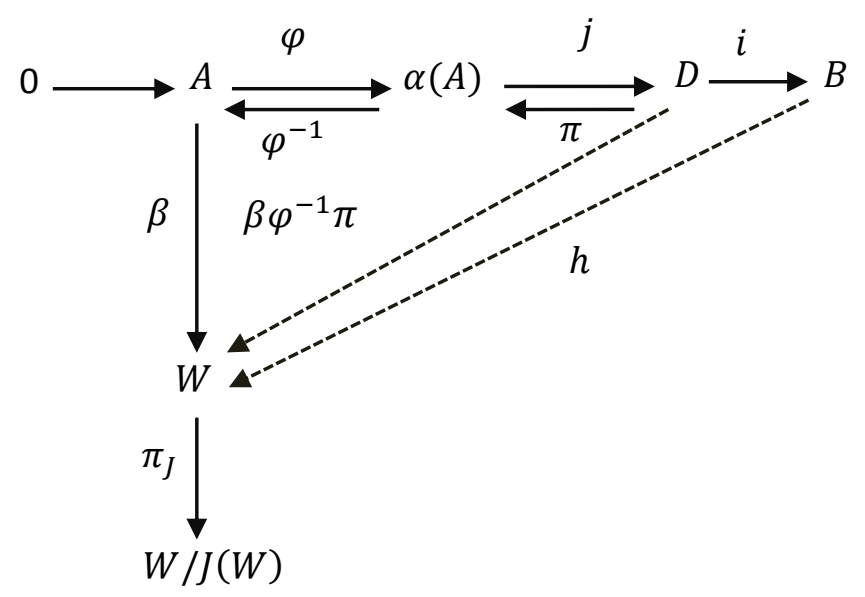

If $\alpha(A)$ is essential in $b$, tnen by (3) there exists $h: B \rightarrow W$ such that $h i=\pi_{J} \beta \varphi^{-1}$ and so $\pi_{J} h i \varphi=\pi_{J} \beta \varphi^{-1} \varphi$ which implies $\pi_{J} h \alpha=\pi_{J} \beta$.

If $\alpha(A)$ is not essential in $B$, then by Lemma (2.20), $D=\alpha(A) \oplus C \preccurlyeq_{e} B$, where $C$ is an inco of $\alpha(A)$ in $B$. Consider the mappings $j: \varphi(A) \rightarrow D$, the natural injection; $\pi: D \rightarrow \varphi(A)$ the natural projection; and $i: D \rightarrow B$, the inclusion map. By (3), there exists $h: B \rightarrow W$ such that $\pi_{J} h i=\pi_{J} \beta \varphi^{-1} \pi$, then $\pi_{J} h i j \varphi=\pi_{J} \beta \varphi^{-1} \pi j \varphi$, note that:

$i j \varphi=\alpha ; \pi j=1_{\varphi(A)} ; \varphi^{-1} \varphi=1_{A}$, so we have $\pi_{J} h \alpha=\pi_{J} \beta$.

Therefore $W$ is nearly injective Ş-semimodule.

Corollary 3.5. An Ş-semimodule $W$ is nearly injective if and only if, for every diagram with $A \preccurlyeq_{e} B$

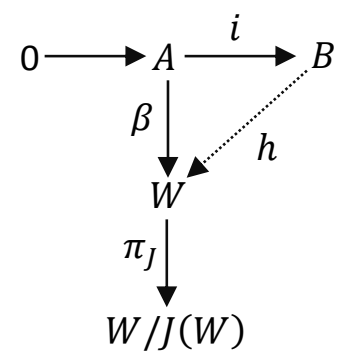

there exists an Ş-homomorphism $h: B \rightarrow W$ such that $\pi_{J} h i=\pi_{J} \beta$, where $\pi_{J}: W \rightarrow$ $W / J(W)$ is the natural projection and $J(W)$ is the Jacobson radical of $W$.

Proposition (3.11) we will give another characterization of nearly injective Şsemimodules by using the class of free semimodules, so we need to mention the following definitions in [12].

Definition 3.6. A set $X$ is called a generated set of the semimodule $A$, if $A$ is the smallest subsemimodule containing $X$, in this case we right $A=\langle X\rangle$.

Remark 3.7. $A=\langle X\rangle$ if and only if $\forall a \in A, a=\sum_{\text {finite }} s_{i} x_{i}, s_{i} \in S_{\supset}, x_{i} \in X$. 
Definition 3.8. A set $A$ is called a free set if for each $\left\{a_{1}, a_{2}, a_{3}, \ldots \ldots \ldots, a_{n}\right\} \subseteq A(n$ a positive integer), the combination $\sum_{i=0}^{n} s_{i} a_{i}=0$ implise $s_{i}=0 \forall i$.

Definition 3.9. A set $A$ is called a basis of the semimodule $W$ if it is a free generating set of $W$.

Definition 3.10. A semimodule $W$ is called a free-semimodule if it has a basis.

Proposition 3.11. An Ş-semimodule $W$ is a nearly injective if and only if, for every diagram with exact row and $B$ is a free Ş-semimodule

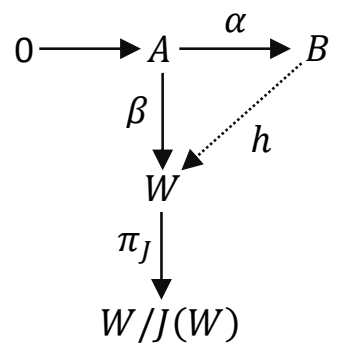

there exists an Ş-homomorphism $h: B \rightarrow W$ such that $\pi_{J} h \alpha=\pi_{J} \beta$, where $\pi_{J}: W \rightarrow$ $W / J(W)$ is the natural projection and $J(W)$ is the Jacobson radical of $W$.

Proof: Necessity is clear.

Sufficiency: Consider the following diagram with $\alpha$ a monomorphism and $F=\oint^{(|B|)}$.

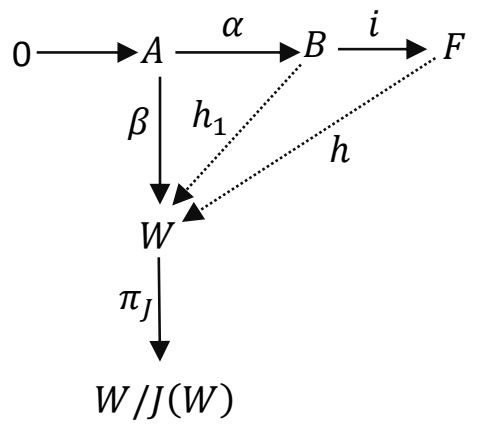

Then $\mathrm{F}$ is a free Ş-semimodule, and $B$ can be considered as a subsemimodule of $F$.

Therefore by hypothesis there exists an Ş-homomorphism $h: F \rightarrow W$ such that $\pi_{J} h i \alpha=\pi_{J} \beta$ ...(i).

So, put $h_{1}=h i: B \rightarrow W$, then we have $\pi_{J} h_{1} \alpha=\pi_{J} h i \alpha$. It follows that by (i) $\pi_{J} h_{1} \alpha=\pi_{J} \beta$.

Hence $W$ is nearly injective Ş-semimodule.

Now, we will study the direct product and the direct sum of nearly injective semimodules. The following propositions shows that this result is true in case of nearly injective semimodules. 
Proposition 3.12. Let $\left\{W_{\lambda}\right\}_{\lambda \in \Lambda}$ be a family of Ş-semimodules and $W=\prod_{\lambda \in \Lambda} W_{\lambda}$, then $W$ is nearly injective if and only if each $W_{\lambda}$ is nearly injective.

Proof: Assume that $W=\prod_{\lambda \in \Lambda} W_{\lambda}$ is nearly injective Ş-semimodules.

Now, we will denote the natural projections, $W$ onto $W / J(W)$ by $\pi_{J}$ and $W_{\lambda}$ onto $W_{\lambda} /$ $J\left(W_{\lambda}\right)$ by $\pi_{J \lambda}$ for any $\lambda \in \Lambda$.

Let $P_{\lambda}: W \rightarrow W_{\lambda}$ and $i_{\lambda}: W_{\lambda} \rightarrow W$ be the projections and injections associated with this direct product respectively for any $\lambda \in \wedge$.

Define $q_{\lambda}: W / J(W) \rightarrow W_{\lambda} / J\left(W_{\lambda}\right)$ by $q_{\lambda}: w+J(W) \mapsto w_{\lambda}+W_{\lambda}$ and

$t_{\lambda}: W_{\lambda} / J\left(W_{\lambda}\right) \rightarrow W / J(W)$ by $t_{\lambda}: w_{\lambda}+W_{\lambda} \mapsto i_{\lambda}\left(w_{\lambda}\right)+J(W)$, then both $q_{\lambda}$ and $t_{\lambda}$ are well defined since $p_{\lambda}(J(W)) \subseteq J\left(W_{\lambda}\right)$ and $\left.i_{\lambda}\left(J\left(W_{\lambda}\right)\right)\right) \subseteq J(W)$ respectively for any $\lambda \in \Lambda$. (it is clear that $p_{\lambda}$ is ? ? ? -regular and $i_{\lambda}$ is an SS-homomorphism. See Proposition (2.29(2)) and the note after it).

Consider the following diagram for any $\lambda \in \wedge$.

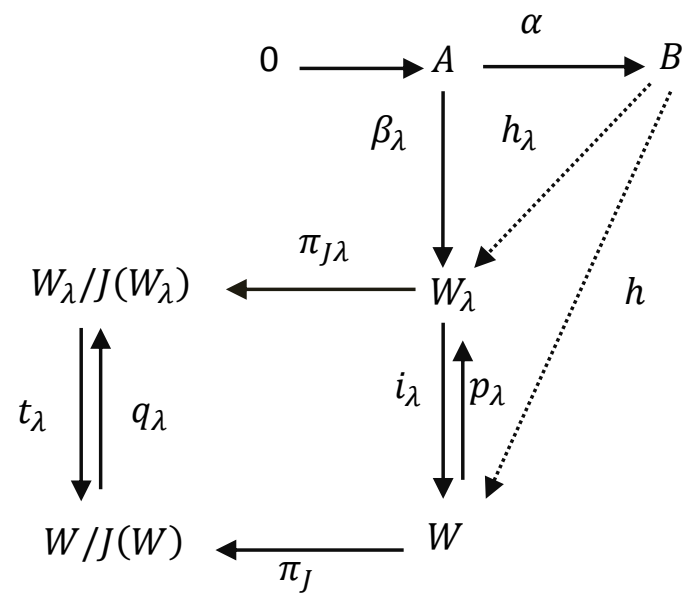

(where $A$ and $B$ be two Ş-semimodules, $u$ a monomorphism and $\beta_{\lambda}$ be an Şhomomorphism )

Note that $\pi_{J \lambda} p_{\lambda}=q_{\lambda} \pi_{J}$

and $\pi_{J} i_{\lambda}=t_{\lambda} \pi_{J \lambda}$

...(ii) and

$q_{\lambda} t_{\lambda}=1_{W_{\lambda} / J\left(W_{\lambda}\right)}$ ...(iii). for any $\lambda \in \wedge$.

So, since $W$ is nearly injective Ş-semimodule then there is an Ş-homomorphism $h: B \rightarrow W$ such that $\pi_{J} h \alpha=\pi_{J} i_{\lambda} \beta_{\lambda}$, for any $\lambda \in \Lambda$ (...(iv).

Now, put $h_{\lambda}=p_{\lambda} h \Rightarrow \pi_{J \lambda} h_{\lambda} \alpha=\pi_{J \lambda} p_{\lambda} h \alpha \Rightarrow \pi_{J \lambda} h_{\lambda} \alpha=q_{\lambda} \pi_{J} h \alpha \quad$ (by (i)).

By (iv) $\pi_{J \lambda} h_{\lambda} \alpha=q_{\lambda} \pi_{J} i_{\lambda} \beta_{\lambda} \Rightarrow \pi_{J \lambda} h_{\lambda} \alpha=q_{\lambda} t_{\lambda} \pi_{J \lambda} \beta_{\lambda} \quad$ (by (ii))

$\Rightarrow \pi_{J \lambda} h_{\lambda} \alpha=1_{W_{\lambda} / J\left(W_{\lambda}\right)} \pi_{J \lambda} \beta_{\lambda}$ (by (iii)) $\Rightarrow \pi_{J} h_{\lambda} \alpha=\pi_{J \lambda} \beta_{\lambda}$ for any $\lambda \in \wedge$. 
Hence $W_{\lambda}$ is nearly injective for any $\lambda \in \Lambda$.

Conversely, assume that $\left\{W_{\lambda}\right\}_{\lambda \in \Lambda}$ is a family of nearly injective Ş-semimodules, for any $\lambda \in \wedge$.

Now, Consider the following diagram with exact row for any $\lambda \in \Lambda$.

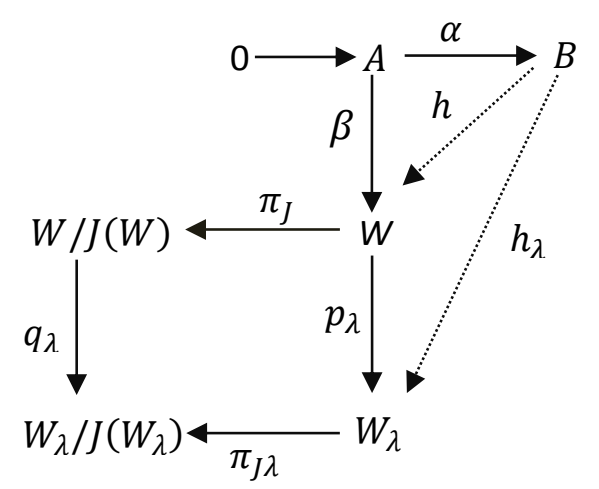

(where $A$ and $B$ be two Ş-semimodules, and $\beta$ be an Ş-homomorphism).

So, since each $W_{\lambda}$ is nearly injective Ş-semimodule, for any $\lambda \in \Lambda$, there is an Şhomomorphism $h_{\lambda}: B \rightarrow W_{\lambda}$ such that $\pi_{J} h_{\lambda} \alpha=\pi_{J} p_{\lambda} \beta$

Now, put $h=\prod_{\lambda \in \Lambda} h_{\lambda}$, then $\pi_{J} h \alpha=\pi_{J} \prod_{\lambda \in \wedge}\left(h_{\lambda} \alpha\right)$

Now, (v) implies $q_{\lambda} \pi_{J} h \alpha=q_{\lambda} \pi_{J} \prod_{\lambda \in \Lambda}\left(h_{\lambda} \alpha\right)$ for each $\lambda$, and then

$\pi_{J \lambda} p_{\lambda} h \alpha=\pi_{J \lambda} p_{\lambda} \prod_{\lambda \in \Lambda}\left(h_{\lambda} \alpha\right)$ for each $\lambda$, but $p_{\lambda} \prod_{\lambda \in \Lambda}\left(h_{\lambda} \alpha\right)=h_{\lambda} \alpha$, and $\pi_{J \lambda} h_{\lambda} \alpha=$ $\pi_{J \lambda} p_{\lambda} \beta$, thus

$\pi_{J \lambda} p_{\lambda} h \alpha=\pi_{J \lambda} p_{\lambda} \beta$ for each $\lambda$. Taking product over $\lambda$ and noticing that $\prod_{\lambda \in \Lambda}\left(\pi_{J \lambda} p_{\lambda}\right)=\pi_{J}$, it follows

$\pi_{J} h \alpha=\pi_{J} \beta$. Therefore $W=\prod_{\lambda \in \wedge} W_{\lambda}$ is nearly injective.

Proposition 3.13. Let $\left\{W_{\lambda}\right\}_{\lambda \in \wedge}$ be a family of Ş-semimodules and $W=\oplus_{\lambda \in \Lambda} W_{\lambda}$, then:

1. If $W$ is nearly injective then each $W_{\lambda}$ is nearly injective.

2. If each $W_{\lambda}$ is nearly injective then $W=\oplus_{\lambda \in \Lambda} W_{\lambda}$ is nearly injective where $\Lambda$ is a finite set.

Proof: (1) The proof is similar to the proof of necessity part of Proposition (3.12).

(2) Clear, since the product and coproduct coincide in the finite case, and by Proposition (3.12). 
Proposition 3.14. Let $\left\{W_{\lambda}\right\}_{\lambda \in \Lambda}$ be a family of Ş-semimodules and $W=\oplus_{\lambda \in \Lambda} W_{\lambda}$, then:

1. If $W / J(W)$ is nearly injective then each $W_{\lambda} / J\left(W_{\lambda}\right)$ is injective for each $\lambda$.

2. If each $W_{\lambda} / J\left(W_{\lambda}\right)$ is nearly injective then $W / J(W)$ is injective, where $\wedge$ is finite set.

Proof: (1) Using the notations of Proposition (3.12), if $\alpha: A \rightarrow B$ is a monomorphism and $\beta: A \rightarrow W_{\lambda} / J\left(W_{\lambda}\right)$ is a homomorphism, since $W / J(W)$ is nearly injective, there is $h: B \rightarrow W / J(W)$ such that $\pi_{J} h \alpha=\pi_{J} t_{\lambda} \beta$, where $\pi_{J}$ is the natural epimorphism of $W / J(W)$ onto $(W / J(W)) / J(W / J(W))$. But $J(W / J(W))=0$, so $\pi_{J}$ can be considered as the identity of $W / J(W)$, hence we have $h \alpha=t_{\lambda} \beta$. Put $h_{\lambda}=q_{\lambda} h$, then $h_{\lambda} \alpha=q_{\lambda} h \alpha=q_{\lambda} t_{\lambda} \beta=1_{W_{\lambda} / J\left(W_{\lambda}\right)} \beta$ (by (iii)) for any $\lambda \in \Lambda \Rightarrow h_{\lambda} \alpha=\beta$.

Therefore, $W_{\lambda} / J\left(W_{\lambda}\right)$ is injective for each $\lambda \in \Lambda$.

(2) Assume that $\left\{W_{k}\right\}(k=1, \ldots, n)$ is a family of Ş-semimodules with $W_{k} / J\left(W_{k}\right)$ is nearly injective for each $k$. Let $\alpha: A \rightarrow B$ be a monomorphism and $\beta: A \rightarrow$ $W / J(W)$ be a homomorphism, using the notations of Proposition (3.12) and since each $W_{k} / J\left(W_{k}\right)$ is nearly injective, there is $h_{k}: B \rightarrow W_{k} / J\left(W_{k}\right)$ such that

$\pi_{k} h_{k} \alpha=\pi_{k} q_{k} \beta$, for $k=1, \ldots, n$, where $\pi_{k}$ is the natural epimorphism of $W_{k} /$ $J\left(W_{k}\right)$ onto $\left(W_{k} / J\left(W_{k}\right)\right) / J\left(W_{k} / J\left(W_{k}\right)\right)$. But $J\left(W_{k} / J\left(W_{k}\right)\right)=0$, so $\pi_{k}$ can be considered as the identity on $W_{k} / J\left(W_{k}\right)$, hence we have

$h_{k} \alpha=q_{k} \beta$, for $k=1, \ldots, n$. Define $h=\sum_{k=1}^{n} t_{k} h_{k}: B \rightarrow W / J(W)$, then $h \alpha=$ $\left(\sum_{k=1}^{n} t_{k} h_{k}\right) \alpha$

$=\left(\sum_{k=1}^{n} t_{k} h_{k} \alpha\right)=\sum_{k=1}^{n} t_{k} q_{k} \beta=\left(\sum_{k=1}^{n} t_{k} q_{k}\right) \beta=1_{W / J(W)} \beta=\beta . \quad$ Therefore $W / J(W)$ is injective.

In the following, the concept of nearly direct summand will be introduced to get a new characterization of nearly injective semimodule.

Definition 3.15. An Ş-subsemimodule $F$ of an Ş-semimodule $W$ is called nearly direct summand of $W$, if for every commutative diagram with exact rows

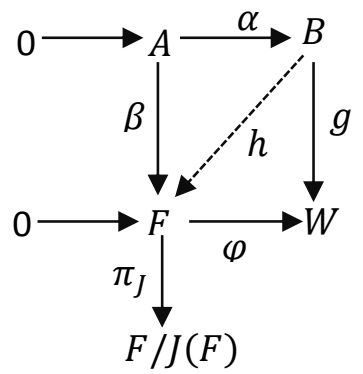

(where $A$ and $B$ be two Ş-semimodules), there is an Ş-homomorphism $h: B \rightarrow F$ such that $\pi_{J} h \alpha=\pi_{J} \beta$, where $\pi_{J}: F \rightarrow F / J(F)$ is the natural epimorphism.

Proposition 3.16. An Ş-subsemimodule $F$ of an Ş-semimodule $W$ is a nearly direct summand of $W$ if and only if, for every diagram with exact row . 


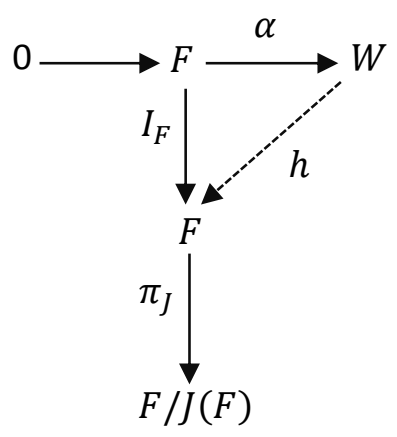

there exists an Ş-homomorphism $h: W \rightarrow F$ such that $\pi_{J} h \alpha=\pi_{J}$, where $\pi_{J}: F \rightarrow$ $F / J(F)$ is the natural epimorphism.

Proof: Assume that $F$ is a nearly direct summand of $W$. Consider the following diagram with exact rows

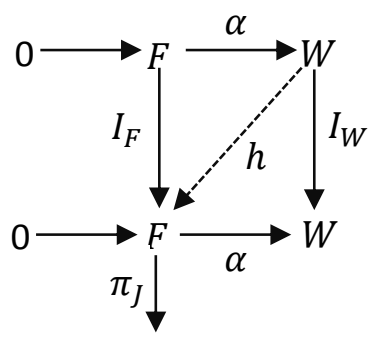

Then by supposition there exists an Ş-hon $\quad F / J(F) \quad h: W \rightarrow F$ such that $\pi_{J} h \alpha=$ $\pi_{J} I_{W}$

This implies $\pi_{J} h \alpha=\pi_{J}$.

Conversely, consider the following commutative diagram with exact rows,

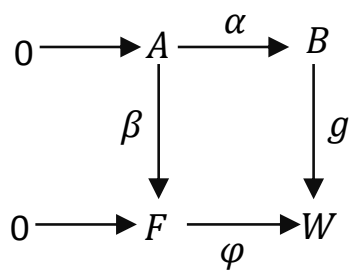

i.e. $g \alpha=\varphi \beta \ldots$... (i). Then we can extend diagram to the following diagram,

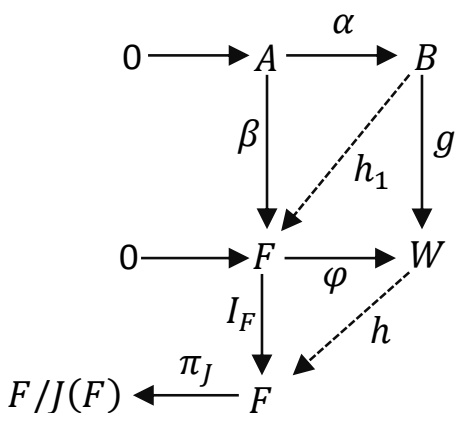


By hypothesis there exists an Ş-homomorphism $h: W \rightarrow F$ such that $\pi_{J} h \varphi=\pi_{J} I_{F}$ ....(ii).

Put $h_{1}=h g: B \rightarrow F$, then we have $\pi_{J} h_{1} \alpha=\pi_{J} h g \alpha$. Then by (i) $\pi_{J} h_{1} \alpha=\pi_{J} h \varphi \beta$.

Hence by (ii) $\pi_{J} h_{1} \alpha=\pi_{J} \beta$. Therefore $F$ is a nearly direct summand of $W$.

The following theorem gives another characterization of nearly injective semimodules.

Theorem 3.17. The following statements are equivalent for an Ş-semimodule $W$ :

1. $W$ is a nearly injective Ş-semimodule.

2. $W$ is a nearly direct summand of every extension of itself.

3. $W$ is a nearly direct summand of every injective extension of itself.

4. $W$ is a nearly direct summand of at least one injective extension of itself.

Proof: $(1) \Rightarrow(2)$ Assume that $W$ is a nearly injective Ş-semimodule .

Let $W_{1}$ be any extension of $W$, consider the following diagram with exact row,

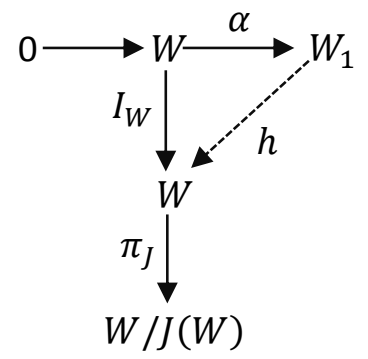

Since $W$ is a nearly injective Ş-homomorphism, there exists an Ş-homomorphism $h: W_{1} \rightarrow W$ such that $\pi_{J} h \alpha=\pi_{J} I_{W} \Rightarrow \pi_{J} h \alpha=\pi_{J}$.

That is $W$ is nearly direct Summand of $W_{1}$ by Proposition (3.16).

$(2) \Longrightarrow(3)$ and $(3) \Rightarrow(4)$ are obvious.

$(4) \Rightarrow$ (1) Assume that $W$ is a nearly direct summand of one injective extension say $W_{1}$.

Consider the following diagram with exact row,

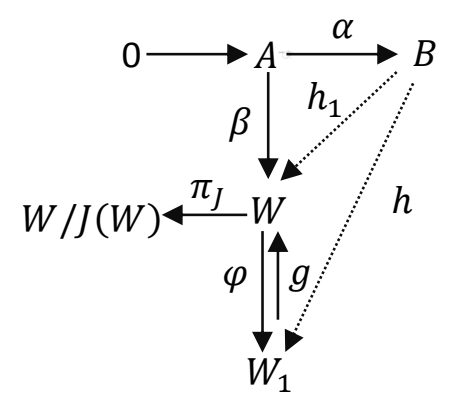


(Where $A$ and $B$ be two Ş-semimodules and $\beta: A \rightarrow W$ be any Ş-homomorphism).

Since $W_{1}$ is an extension of $W$ thus, there is an Ş-monomorphism, say $\varphi: W \rightarrow W_{1}$.

By injectivity of $W_{1}$, there exists an Ş-homomorphism $h: B \rightarrow W_{1}$ such that $h \alpha=$ $\varphi \beta$

Now, since $W$ is a nearly direct summand of $W_{1}$, thus there exists an Şhomomorphism. $g: W \rightarrow W_{1}$ such that $\pi_{J} g \varphi=\pi_{J}$ (3.16)).

...(ii) (by Proposition

Put $h_{1}=g h: B \rightarrow W$, then we have $\pi_{J} h_{1} \alpha=\pi_{J} g h \alpha \Rightarrow$ by (i) $\pi_{J} h_{1} \alpha=\pi_{J} g \varphi \beta$

Hence by (ii) $\pi_{J} h_{1} \alpha=\pi_{J} \beta$. Therefore $W$ is nearly injective Ş-semimodule.

Corollary 3.18. Let $W$ be a $\mathcal{Y C} \mathcal{S}$-semimodule which is contained in an injective Şsemimodule, then $W$ is nearly injective if and only if $W$ is a nearly direct summand of $E(W)$ (where $E(W)$ is the injective envelope of $W$ ).

Proof: Since $W$ is contained in an injective Ş-semimodule, then it has an injective envelope, say $E(W)$. The proof follows from the equivalence of (1) and (4) of Theorem 3.17.

In the following, the concept of nearly split will be introduced to get a new characterization of nearly injective semimodule.

Definition 3.19. Let $A$ and $B$ be two Ş-semimodules. An Ş-homomorphism $\alpha: A \rightarrow B$ is called nearly split if there is an Ş-homomorphism $\beta: B \rightarrow A$ such that $\pi_{J} \beta \alpha=\pi_{J}$, where $\pi_{J}: W \rightarrow W / J(W)$ is the natural epimorphism.

The following theorem gives another characterization of nearly injective semimodules.

Theorem 3.20. Let $W$ be a $\mathcal{Y C S}$-semimodule which is contained in an injective Şsemimodule, then the following statements are equivalent for an Ş-semimodule $W$ :

1. $W$ is a nearly injective Ş-semimodule.

2. For each Ş-semimodule $F$, every Ş-monomorphism $\alpha: W \rightarrow F$ is a nearly split.

3. For each nearly injective Ş-semimodule $F$, every Ş-monomorphism $\alpha: W \rightarrow F$ is a nearly split.

4. For each injective Ş-semimodule $F$, every Ş-monomorphism $\alpha: W \rightarrow F$ is a nearly split.

5. Every S-monomorphism $\alpha: W \rightarrow E(W)$ is nearly split (where $E(W)$ is the injective envelope of $W$.

Proof: $(1) \Longrightarrow(2)$ Assume that $W$ is nearly injective Ş-semimodule and $\alpha: W \rightarrow F$ be any S-monomorphism (where $F$ is any S-semimodule. Consider the following diagram, 


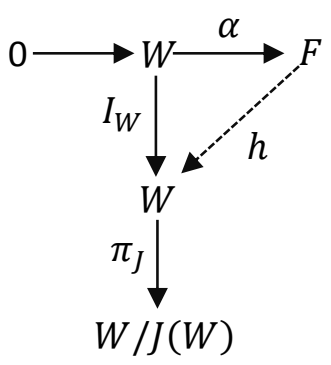

Since $W$ is nearly injective, thus there exists an Ş-homomorphism $h: F \rightarrow W$ such that

$\pi_{J} h \alpha=\pi_{J} I_{W}=\pi_{J}$. Therefore $\alpha$ is nearly split by Definition (3.19).

$(2) \Longrightarrow(3),(3) \Rightarrow(4)$ and $(4) \Rightarrow(5)$ are obvious.

$(5) \Rightarrow(1)$ Assume $\alpha: W \rightarrow E(W)$ is a nearly split.

Since $E(W)$ is an extension of $W$ by Proposition (2.25), thus there exists an Şmonomorphism, say $\alpha: W \rightarrow E(W)$. Consider the following commutative diagram with exact row,

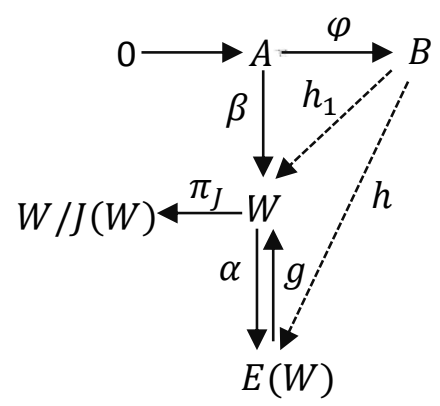

Now, by injectivity of $E(W)$, there exists an Ş-homomorphism. $h: B \rightarrow E(W)$ such that $h \varphi=\alpha \beta \quad$...(i). But by assumption $\alpha: W \rightarrow E(W)$ is nearly split, then there is an Ş-homomorphism. $g: E(W) \rightarrow W$ such that $\pi_{J} g \alpha=\pi_{J}$

So, put $h_{1}=g h: B \rightarrow W$, then we have $\pi_{J} h_{1} \varphi=\pi_{J} g h \varphi$. It follows by (i) $\pi_{J} h_{1} \varphi=$ $\pi_{J} g \alpha \beta \Rightarrow \pi_{J} h_{1} \varphi=\pi_{J} \beta$ by (ii). Hence $W$ is nearly injective Ş-semimodule. 


\section{CONFLICT OF INTERESTS.}

There are non-conflicts of interest.

\section{References}

1. H. M. J. Al-thani, "Projective and Injective Semimodules over Semirings", .Ph.D. dissertation, East London Univ., 1998.

2. A. R. Mehdi, "Nearly injective modules", M.S. thesis, Univ. of Al-Mustansiriya, Baghdad, Iraq, 2000.

3. J. R. Tsiba and D. Sow, "On Generators and Projective Semimodules", International Journal of Algebra, vol. 4, no. 24, pp.1153-1167, Sep. 2010.

4. J. S. Golan, "Semiring and their Aplications", Springer Science and Business media, , London, 2013.

5. A. M. Alhossaini and Z. A. Aljebory, "On p-duo Semimodules", Journal of University of Babylon, vol. 26, no. 4, 2018.

6. F. Kasch, "Modules and Rings", Academic press, London, New York, 1982.

7. K. Pawar, "A Note on Essential Subsemimodules", New Trends in Mathematical Sciences, vol. 1, no. 2, pp.18-21, 2013.

8. D. W. Sharpe and P. Vamos, "Injective Modules", Cambridge Univ. press, London, 1972.

9. S. N. Il'in, “On injective envelopes of semimodules over semirings", Journal of Algebra and Its Applications, vol. 15, no. 6, pp.1-13, Aug. 2015.

10. A. M. Alhossaini and S. H. Alsaebari, "Preradical on semimodules", Baghdad Science Journal, to appear.

11. N.X. Tuyen and H.X Thang, "On Superfluous Subsemimodules". Georgian Mathematical Journal. vol. 10, no. 4, pp.763-77, 2003.

12- A. M. Alhossaini and Z. A. Aljebory, "Fully Dual Stable Semimodule", Journal of Iraqi Al-khwarizmi, vol. 1, no. 1, pp.92-100, 2017.

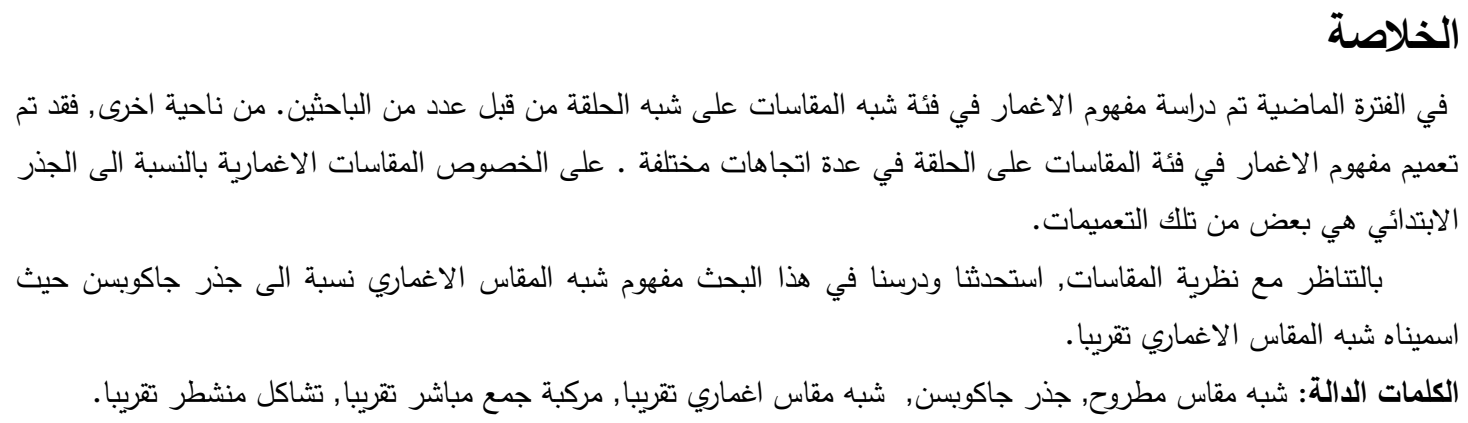

